\title{
BUENAS PRÁCTICAS PARA LA REALIZACIÓN DE MOVILIDAD DE ESTUDIANTES Y DOCENTES INVESTIGADO- RES DE LA FUNDACIÓN UNIVERSITARIA SAN MATEO
}

\author{
Merly María Bernal Llanos \\ Programa de Negocios internacionales, \\ Relacionista Económica Internacional. \\ Correo electrónico: mmbernal@funsanmateo.edu.co
}

\section{Resumen}

Con la investigación se pretende verificar el cumplimiento de las buenas prácticas en la realización de movilidad de investigadores de la Fundación Universitaria San Mateo e identificar los inconvenientes que tienen estudiantes y docentes para pertenecer a los grupos existentes, llámese semillero, ayudantías, pasantías o trabajos de grado; de igual manera, busca que se unan cada vez más la academia, la investigación y la práctica profesional que contribuya a la participación activa, fortaleciendo las actuales líneas y grupos de investigación.

\section{Introducción}

El presente documento busca analizar y mejorar la relación que existe actualmente entre la investigación y la academia, al mismo tiempo que se propongan estrategias que involucren a docentes y estudiantes en la participación de las líneas y grupos existentes en la Fundación Universitaria San Mateo.

La investigación que se realiza es de alcance descriptivo, enfoque cualitativo, de diseño no experimental y clasificado transeccional o transversal. Se desarrolla en etapas: primera fase o estado del arte; segunda fase o de trabajo de campo (encuesta) para aplicar a estudiantes y docentes; tercera fase o de procesamiento de datos y la cuarta fase llevar de elaboración de un Working papper.

Así mismo, mediante análisis de los resultados que se obtienen de las encuestas ejecutadas, se puede identificar el impacto real que tiene la investigación dentro de la Institución.

Las personas interesadas en participar en investigación deben revisar el manual de instrucciones para movilidad de docentes y estudiantes investigadores, el cual es de utilidad antes de presentar proyectos investigativos, ponencias y artículos, garantizando la calidad de sus procesos. 


\section{Contenido}

En Colombia existen instituciones del gobierno como el Ministerio de Educación Nacional (MEN) y el Consejo Nacional de Acreditación (CNA), quienes estiman que la visibilidad a nivel nacional e internacional de las universidades y los programas ofertados, son un factor importante para la acreditación de las mismas y que conllevan a la creación de convenios de cooperación o integración entre Instituciones en áreas del quehacer académico, investigativo, tecnológico y científico.

Los integrantes de grupos de investigación son los que hacen parte de esta actividad y sus hojas de vida deben estar incluidas en el CVLAC. Las cuatro clasificaciones son: investigadores, investigadores en formación, estudiantes de pregrado e integrante vinculado.

De acuerdo con la convocatoria 781 de 2017 denominada "Convocatoria Nacional para el Reconocimiento y Medición de Grupos de Investigación, Desarrollo Tecnológico o de Innovación y para el Reconocimiento de Investigadores del SNCTel, 2017", los resultados que obtuvo la Fundación Universitaria San Mateo fueron:

\begin{tabular}{|c|c|c|c|c|c|c|}
\hline \multirow[b]{2}{*}{$\begin{array}{l}\text { CODIGO DE } \\
\text { GRUPO }\end{array}$} & \multicolumn{2}{|c|}{ (90) COLCIENCIAS } & \multicolumn{2}{|c|}{ (2) GOBIERNO DE COLOMBIA } & & \\
\hline & NOMBRE DEL GRUPO & $\begin{array}{l}\text { LIDER DEL } \\
\text { GRUPO }\end{array}$ & $\begin{array}{l}\text { INSTITUCIONES } \\
\text { QUE LO AVALAN }\end{array}$ & $\begin{array}{c}\text { GRUPO } \\
\text { RECONOCIDO }\end{array}$ & $\begin{array}{l}\text { GRUPO } \\
\text { INSCRITO } \\
\text { PARA } \\
\text { MEDICIÓN }\end{array}$ & $\begin{array}{l}\text { CLASIFICACIÓN } \\
\text { DEL GRUPO }\end{array}$ \\
\hline$\underline{\text { COL0109972 }}$ & $\begin{array}{l}\text { GESTIONNY DESARRO- } \\
\text { LLO DE SOLUCIONES } \\
\text { TECNOLOGICASY DE } \\
\text { INGENIERIAPARALAS } \\
\text { ORGANIZACIONES }\end{array}$ & $\begin{array}{l}\text { RICHAR ALBER- } \\
\text { TO RANGEL } \\
\text { MARTINEZ }\end{array}$ & $\begin{array}{l}\text { FUNDACION } \\
\text { PARALAEDUCA- } \\
\text { CION SUPERIOR } \\
\text { SAN MATEO } \\
\end{array}$ & $\checkmark$ & . & c \\
\hline COL0109981 & $\begin{array}{l}\text { FUS EMPRESAS: INNO- } \\
\text { VACION Y DESARROLLO } \\
\text { CREATIVO } \\
\end{array}$ & $\begin{array}{l}\text { SONIAESME- } \\
\text { RALDAROJAS } \\
\text { ROJAS } \\
\end{array}$ & $\begin{array}{l}\text { FUNDACION } \\
\text { PARALAEDUCA- } \\
\text { CION SUPERIIR } \\
\text { SAN MATEO } \\
\end{array}$ & $\checkmark$ & si & c \\
\hline COL0161121 & $\begin{array}{l}\text { PATRIMONIO CULTURAL } \\
\text { Y GASTRONOMICO DE } \\
\text { COLOMBIA } \\
\end{array}$ & $\begin{array}{l}\text { ELIZABETH } \\
\text { ARAQUE ELAICA }\end{array}$ & $\begin{array}{l}\text { FUNDACION } \\
\text { PARALAEDUCA- } \\
\text { CION SUPERIOR } \\
\text { SAN MATEO } \\
\end{array}$ & $\checkmark$ & si & c \\
\hline COL0173178 & $\begin{array}{c}\text { BIMAT BUSSINESS IN- } \\
\text { TERNATIONALL MARKET } \\
\text { TRADE } \\
\end{array}$ & $\begin{array}{l}\text { MARTHA MILENA } \\
\text { CUELLAR CHA. } \\
\text { VES }\end{array}$ & $\begin{array}{l}\text { FUNDACION } \\
\text { PARALAEDUCA- } \\
\text { IION SUPERIOR } \\
\text { SAN MATEO }\end{array}$ & $\checkmark$ & si & c \\
\hline COL0173294 & $\begin{array}{l}\text { ESTUDIOS DE LA IMA- } \\
\text { GEN }\end{array}$ & \begin{tabular}{|c|} 
OMAR ALONSO \\
GARCIA MARTi- \\
NEZ
\end{tabular} & $\begin{array}{l}\text { FUNDACION } \\
\text { PARALAEDUCA- } \\
\text { CION SUPERIOR } \\
\text { SAN MATEO }\end{array}$ & $\checkmark$ & si & Reconocido \\
\hline
\end{tabular}

(Figura 1)

Figura 1. Convocatoria para el Reconocimiento y Medición de Grupos de Investigación. Fuente: Colciencias 2017

Con lo anterior, se entiende que las actividades que lleva a cabo una Institución de Educación Superior (en adelante IES) son importantes y se tienen en cuenta para la acreditación de los programas ofertados, fortaleciendo la identidad de la Institución y el reconocimiento a nivel local, nacional e internacional. Actualmente dos puntos dentro de la visita de pares académicos que han adquirido mayor relevancia es la investigación y la generación permanente de intercambios de docentes y estudiantes.

La investigación es la generación o producción de conocimiento, por lo tanto, las IES deben formar capital humano para desarrollar actividades científicas de calidad que permitan aumentar el dinamismo en dicha gestión. La parte administrativa también debe estar involucrada en el proceso con el fin que docentes y estudiantes presenten y lleven a cabo proyectos investigativos con recursos financiados por las Instituciones.

Con la elaboración de documentos investigativos, artículos y con asesoría por parte de los coordinadores encargados del tema, se contribuye a la formación de investigadores calificados, que mejoran y fortalecen la capacidad de las personas que participan en esta actividad, que a su vez aumenta la generación de nuevo conocimiento y facilita la 
representación de la institución con ponencias en los diferentes congresos a los que se postulen, motivando la movilidad académica e integrando conocimiento, cultura, desarrollo social y tecnológico.

La Fundación Universitaria San Mateo cuenta con documentos que ayudan para que la investigación adquiera mayor importancia en la Institución y aumente la clasificación en Colciencias, ofreciendo información de calidad que contribuya al conocimiento de la sociedad en general.

\section{Estatuto Investigación}

Propuesta institucional fundamental en la formación de profesionales idóneos que aporten al desarrollo de la región y el país, desde la ciencia, la tecnología, la investigación y la cultura; refiriendo todos los ámbitos del conocimiento: ciencias exactas, socio-histórico, humanísticas, artísticas; bajo los principios de integralidad e interdisciplinariedad como estrategia de retroalimentación de los procesos académicos, con espíritu ético, cívico, creativo y crítico (Fundación para la Educación Superior San Mateo, 2012, pág. 7).

Para dar cumplimiento con lo anterior, la Fundación Universitaria San Mateo cuenta con los siguientes semilleros de investigación, dirigidos por los coordinadores de programa:

\begin{tabular}{|c|l|l|}
\hline No. & Semillero de investigación & Programa \\
\hline 1 & Semillero de Inteligencia de Negocios & Ingeniería de Sistemas \\
\hline 2 & Semillero de Desarrollo de Software & Ingeniería de Sistemas \\
\hline 3 & Semillero de Realidad Aumentada & Ingeniería de Sistemas \\
\hline 4 & Semillero de Transportes Inteligentes & $\begin{array}{l}\text { Ingeniería en } \\
\text { Telecomunicaciones }\end{array}$ \\
\hline 5 & Semillero en Robótica & $\begin{array}{l}\text { Ingeniería en } \\
\text { Telecomunicaciones }\end{array}$ \\
\hline 6 & Semillero de Redes Satelitales & $\begin{array}{l}\text { Ingeniería en } \\
\text { Telecomunicaciones }\end{array}$ \\
\hline 7 & Semillero Patrimonio Cultural y Gastronómico & Gastronomía \\
\hline 8 & Semillero BIMAT Negocios & Negocios Internacionales \\
\hline 9 & Semillero Mapeo del TLC Negocios & Negocios Internacionales \\
\hline 10 & Semillero Contaduría -SIC- & Contaduría Pública \\
\hline 11 & Semillero en Procesos y Sistemas Sostenibles & $\begin{array}{l}\text { Ingeniería Industrial - Ingenie- } \\
\text { ría Seguridad y Salud para } \\
\text { el Trabajo }\end{array}$ \\
\hline 12 & $\begin{array}{l}\text { Semillero en Condiciones de Salud y Trabajo } \\
\text { de Diferentes Ocupaciones }\end{array}$ & $\begin{array}{l}\text { Ingeniería Industrial - Ingenie- } \\
\text { ría en Seguridad y Salud para el } \\
\text { Trabajo }\end{array}$ \\
\hline 13 & Semillero en Administración & Administración de Empresas \\
\hline 14 & Semillero en Diseño Gráfico & Diseño Gráfico \\
\hline
\end{tabular}

Figura 2. Merly María Bernal Llanos Fuente: autoría propia 
Los estudiantes cuentan con diferentes alternativas para graduarse, no obstante, las que competen a investigación son:

\section{Trabajo de grado}

- Asociados a las líneas institucionales de investigación.

- Institucional (Fundación Universitaria San Mateo con otras instituciones afines).

- Interinstitucional (aporte al desarrollo local, nacional e internacional).

- Otras modalidades de trabajos que el comité curricular y de investigación de los Programas consideren oportunos.

\section{Pasantía de investigación}

- Estudio de investigación que busca la solución a un problema empresarial del sector público o privado.

- Fortalecer perfil académico y profesional de los participantes.

- Retroalimentación del plan de estudio de acuerdo a las necesidades del medio.

\section{Ayudantía de investigación}

- Vinculación de estudiantes de últimos semestres (ciclos propedéuticos) a investigaciones de docente de los programas (Proyecto Institucional) o vinculación a proyectos externos.

- Recolectar información a través de la internet, visitas a empresas, IES u otras que requiera la investigación.

- Procesar la información y entregar reportes semanales para luego reportar a la coordinación de investigación.

- Verificación de fuentes o recursos bibliográficos.

- De manera presencial, distancia y virtual.

La unidad ejecutiva, académica y administrativa responsable de la coordinación, promoción, asesoría, gestión y regulación de los diferentes componentes del Sistema Institucional de la Investigación de la Fundación Universitaria San Mateo es el Centro de Desarrollo Tecnológico y sus siglas CDTEC (actualmente dirección de investigaciones) (Fundación para la Educación Superior San Mateo, 2012, pág. 39).

De igual manera, la Fundación Universitaria San Mateo cuenta con un reglamento de propiedad intelectual para que ninguno de sus investigadores tenga inconvenientes con sus escritos (investigación, artículos, ensayos y demás). El documento se encuentra en la página institucional en el ícono de investigación y dentro del mismo se puede desplegar una serie de opciones de interés:

- Semilleros

- Documentos Importantes

- Opciones de Grado

- ¿Quiénes somos?

- Grupos de Investigación

- Banco de Proyectos

El estudiante, docente, administrativo y personal externo podrá consultar libremente la página y encontrar "grosso modo" lo que necesite para cumplir con los requerimientos de investigación. 


\section{Método}

Para el desarrollo de la investigación, el estudio es de alcance descriptivo, porque "busca especificar las propiedades y características importantes de cualquier fenómeno que se analice. Describe tendencias de un determinado grupo o población" (Hernández Sampieri, R.; Fernández Collado, C.; y Baptista Lucio, M., 2014 pág 92), pretende recolectar y medir información de forma independiente a las variables establecidas más no como se relacionan entre ellas.

Por consiguiente, se describirán con precisión las etapas representativas de la investigación: buenas prácticas para la realización de movilidad de estudiantes y docentes investigadores de la Fundación Universitaria San Mateo.

Por otro lado, es de diseño no experimental puesto que es un "estudio que se realiza sin la manipulación deliberada de variables y en los que solo se observan los fenómenos en su ambiente natural para analizarlos" (2014, pág 152), clasificado en diseño transeccional o transversal, porque recolecta datos en un solo momento, "su propósito es describir variables y analizar su incidencia e interrelación en un momento dado" (2014, pág. 154).

La investigación se divide en las siguientes fases: La primera fase o estado del arte; segunda fase o de trabajo de campo (encuesta) para aplicar a estudiantes y docentes; tercera fase o de procesamiento de datos y la cuarta fase llevar de elaboración de un Working papper.

En el trabajo de campo, se diseña una encuesta que se aplica a 100 personas de la Institución, especialmente a estudiantes y docentes.

\section{Análisis}

Del análisis de la información recopilada a través de encuesta realizada a 100 personas de la Institución, especialmente a estudiantes y docentes:

La pregunta del rol de investigador y programa académico indica que el 85\% de los encuestados son estudiantes, el $8 \%$ docente y el 7\% dice que ninguno de los dos roles. Con relación a los programas académicos que participan, entre docentes y estudiantes el 38\% dice que pertenecen a negocios internacionales, el 17\% no quiso decir a qué programa pertenece, el 14\% al programa de contaduría pública, el 12\% a administración de empresas, el 10\% a gastronomía y el 9\% a ingeniería de telecomunicaciones.

\begin{tabular}{|c|c|c|c|}
\hline \multicolumn{2}{|c|}{ ¿Su rol de investigador es? } & Respuesta & Porcentaje \\
\cline { 2 - 4 } & Docente & 8 & $8 \%$ \\
\hline Estudiante & 85 & $85 \%$ \\
\cline { 2 - 4 } & Ninguno de los dos & 7 & $7 \%$ \\
\cline { 2 - 4 } (Tabla 1) & TOTAL & 100 & $100 \%$ \\
\hline
\end{tabular}

Tabla 1. Rol del investigador 


\begin{tabular}{|c|c|c|}
\hline ¿Su rol de investigador es? & Respuesta & Porcentaje \\
\hline Docente & 8 & $8 \%$ \\
\hline Estudiante & 85 & $85 \%$ \\
\hline Ninguno de los dos & 7 & $7 \%$ \\
\hline TOTAL & 100 & $100 \%$ \\
\hline
\end{tabular}

(Tabla 1)

Tabla 1. Rol del investigador

\begin{tabular}{|c|c|c|}
\hline Programa & Respuesta & Porcentaje \\
\hline No quiso decir a qué programa pertenece & 17 & $17 \%$ \\
\hline Negocios Internacionales & 38 & $38 \%$ \\
\hline Contaduría Pública & 14 & $14 \%$ \\
\hline Administración de Empresas & 12 & $12 \%$ \\
\hline Gastronomía & 10 & $10 \%$ \\
\hline Ingeniería de Telecomunicaciones & 9 & $9 \%$ \\
\hline TOTAL & 100 & $100 \%$ \\
\hline
\end{tabular}

(Tabla 1.1)

Tabla 1.1 Programa académico Fuente: elaboración propia

Dentro de la Institución ubica (si/no) la oficina del CDTEC (o dirección de investigaciones), dice que el $79 \%$ conoce y el $21 \%$ no la conoce.

\begin{tabular}{|c|c|c|}
\hline $\begin{array}{c}\text { Dentro de la Institución. } \\
\text { ¿Ubica la Oficina del } \\
\text { CDTEC? }\end{array}$ & Respuesta & Porcentaje \\
\hline Sí & 21 & $21 \%$ \\
\hline No & 79 & $79 \%$ \\
\hline TOTAL & 100 & $100 \%$ \\
\hline
\end{tabular}

Tabla 2. Oficina CDTEC Fuente: elaboración propia 
De acuerdo con la pregunta si conoce al director de la oficina del CDTEC y sus colaboradores, el $80 \%$ indica que no conoce al director de la oficina del CDTEC y el $20 \%$ sí lo conoce. De igual manera, se indica que el 20\% sí conoce y el 80\% responde que no conoce a los colaboradores del CDTEC.

\begin{tabular}{|c|c|c|c|}
\hline $\begin{array}{c}\text { ¿Conoce al Director de la } \\
\text { Oficina del CDTEC? }\end{array}$ & Respuesta & Porcentaje \\
\hline Sí & 20 & $20 \%$ \\
\hline No & 80 & $80 \%$ \\
\hline (Tabla 3) & TOTAL & 100 & $100 \%$ \\
\hline
\end{tabular}

Tabla 3. Director del CDTEC Fuente: elaboración propia

\begin{tabular}{|c|c|c|c|}
\hline & $\begin{array}{c}\text { ¿Conoce a los } \\
\text { colaboradores oficina } \\
\text { CDTEC? }\end{array}$ & Respuesta & Porcentaje \\
\hline & Sí & 20 & $20 \%$ \\
\hline \multirow{2}{*}{ (Tabla 3.1) } & No & 80 & $80 \%$ \\
\hline & TOTAL & 100 & $100 \%$ \\
\hline
\end{tabular}

Tabla 3.1 Colaboradores del CDTEC Fuente: elaboración propia

La respuesta a la pregunta de si identifica al coordinador de investigación de su programa, indica que $64 \%$ de los estudiantes de la Institución no los conoce y el otro 36\% dice que sí los conoce.

\begin{tabular}{|c|c|c|c|}
\hline \multirow{4}{*}{ (Tabla 4) } & $\begin{array}{c}\text { ¿Conoce a los } \\
\text { colaboradores oficina } \\
\text { CDTEC? }\end{array}$ & Respuesta & Porcentaje \\
\hline & Sí & 20 & $20 \%$ \\
\hline & No & 80 & $80 \%$ \\
\hline & TOTAL & 100 & $100 \%$ \\
\hline
\end{tabular}

Tabla 4. Coordinador de investigación de su Programa Fuente: elaboración propia 
El porcentaje de respuestas a la pregunta si conoce los requisitos para realizar investigaciòn dentro de la Institución es que el 76\% de los estudiantes encuestados no los conoce y el $24 \%$ dice que sí los conoce.

\begin{tabular}{|c|c|c|c|}
\hline \multirow{4}{*}{$\begin{array}{c}\text { ¿Conoce los requisitos para } \\
\text { realizar investigación en la } \\
\text { San Mateo? }\end{array}$} & Respuesta \\
\cline { 2 - 4 } & Sí & 24 & $24 \%$ \\
\cline { 2 - 4 } & No & 76 & $76 \%$ \\
\cline { 2 - 4 } & TOTAL & 100 & $100 \%$ \\
\hline
\end{tabular}

Tabla 5. Requisitos para realizar investigación Fuente: elaboración propia

| Los resultados a la pregunta de si ha presentado proyectos de investigaciòn ante el CDTEC, el 76\% de los encuestados dice que no han presentado Proyectos de Investigaciòn ante el CDTEC y el 24\% indica que sí lo ha hecho.

\begin{tabular}{|c|c|c|c|}
\hline \multirow{4}{*}{$\begin{array}{c}\text { ¿Ha presentado proyecto } \\
\text { de investigación ante el } \\
\text { CDTEC? }\end{array}$} & Respuesta & Porcentaje \\
\cline { 2 - 4 } (Tabla 6) & Sí & 24 & $24 \%$ \\
\cline { 2 - 4 } & No & 76 & $76 \%$ \\
\cline { 2 - 4 } & TOTAL & 100 & $100 \%$ \\
\hline
\end{tabular}

Tabla 6. Proyectos de Investigación Fuente: elaboración propia

Con la respuesta de si ha participado con su investigación en congresos nacionales e Internacionales, se puede observar que el $84 \%$ de los encuestados no ha participado en congresos de investigación, mientras que el 16\% sí lo ha hecho. De igual manera, se indica que el $85 \%$ de los encuestados no ha participado en congresos a nivel nacional e internacional, el 10\% lo ha hecho a nivel nacional y el 5\% a nivel internacional.

\begin{tabular}{|c|c|c|c|}
\hline \multirow{4}{*}{$\begin{array}{c}\text { ¿Con su investigación ha } \\
\text { participado en congresos } \\
\text { de investigación? }\end{array}$} & Respuesta & Porcentaje \\
\cline { 2 - 4 } Sí & 16 & $16 \%$ \\
\cline { 2 - 4 } & No & 84 & $84 \%$ \\
\hline TOTAL & 100 & $100 \%$ \\
\hline
\end{tabular}

Tabla 7. Participación en Congresos de Investigaciòn, nacional e internacional Fuente: elaboración propia 


\begin{tabular}{|c|c|c|c|}
\hline & $\begin{array}{c}\text { ¿Con su investigación ha } \\
\text { participado en congresos } \\
\text { de investigación? }\end{array}$ & Respuesta & Porcentaje \\
\hline \multirow{3}{*}{ (Tabla 7) } & Sí & 16 & $16 \%$ \\
\hline & No & 84 & $84 \%$ \\
\hline & TOTAL & 100 & $100 \%$ \\
\hline
\end{tabular}

Tabla 7. Participación en Congresos de Investigaciòn, nacional e internacional Fuente: elaboración propia

La respuesta de si conoce el procedimiento para realizar movilidad en investigación en la Institución, resume que el 92\% de las personas encuestadas no, el 8\% restante indica que sí lo conoce.

\begin{tabular}{|c|c|c|c|}
\hline \multirow{4}{*}{$\begin{array}{c}\text { Conoce el procedimiento para } \\
\text { realizar movilidad en investigación } \\
\text { de la San Mateo? }\end{array}$} & Respuesta & Porcentaje \\
\cline { 2 - 4 } (Tála 8) & Sí & 82 & $8 \%$ \\
\cline { 2 - 4 } & No & 100 & $92 \%$ \\
\hline TOTAL & $92 \%$ \\
\hline
\end{tabular}

Tabla 8. Procedimiento movilidad en investigación Fuente: elaboración propia

La respuesta a la pregunta de si considera que hacer investigación en la Institución le sirve para su área académica, el 67\% dice que sí y el 33\% indica que no le sirve. A| la respuesta del ¿por qué?, el 30\% dice que mejora el análisis e interpretación de información, el 25\% adquiere conocimiento profundo, el 23\% mejora la academia, el 15\% que con ella puede graduarse y el $7 \%$ restante no sabe, no responde.

\begin{tabular}{|c|c|c|c|}
\hline \multirow{4}{*}{ (Tabla 9) } & $\begin{array}{c}\text { ¿Considera que hacer investiga- } \\
\text { ciòn en la San Mateo le sirve para } \\
\text { su área académica? }\end{array}$ & Respuesta & Porcentaje \\
\hline & Sí & 67 & $67 \%$ \\
\hline & No & 33 & $33 \%$ \\
\hline & TOTAL & 100 & $100 \%$ \\
\hline
\end{tabular}

Tabla 9. Investigación en la San Mateo y el área académica Fuente: elaboración propia 


\begin{tabular}{|c|c|c|c|}
\hline \multirow{1}{*}{ (Tabla 9.1) } & Respuesta & Porcentaje \\
\cline { 2 - 4 } & $\begin{array}{c}\text { No sabe no responde } \\
\text { pretación de información }\end{array}$ & 7 & $7 \%$ \\
\cline { 2 - 4 } & $\begin{array}{c}\text { Conocimiento más } \\
\text { profundo }\end{array}$ & 30 & $30 \%$ \\
\hline Para poder graduarme & 25 & $25 \%$ \\
\hline Mejora la academia & 15 & $15 \%$ \\
\hline TOTAL & 23 & $23 \%$ \\
\hline
\end{tabular}

Tabla 9.1 ¿Por qué?

Fuente: elaboración propia

Con base en la respuesta ¿Cómo ha sido su experiencia al representar a la Institución en movilidad de investigación a nivel local, nacional e internacional?, se observa que el 50\% de encuestados no la ha representado en dicho campo, el 15\% lo considera una buena opción de grado, conocen otras maneras de investigar, otro 15\% dice que es bueno mejorar el sistema que tienen para aumentar cantidad y calidad en investigaciones, un 10\% indica que es muy interesante, gratificante, amplía el conocimiento y el último 10\% dice que tiene conocimiento cultural en todo el sentido de la palabra.

\begin{tabular}{|c|c|c|c|}
\hline \multirow{7}{*}{ (Tabla 10) } & $\begin{array}{c}\text { ¿Cómo ha sido su experiencia al representar a } \\
\text { la Institución en movilidad de investigación a } \\
\text { nivel local, nacional e internacional? }\end{array}$ & Respuesta & Porcentaje \\
\hline & N/A & 50 & $50 \%$ \\
\hline & $\begin{array}{l}\text { Muy Interesante, Gratificante, amplía el conoci- } \\
\text { miento }\end{array}$ & 10 & $10 \%$ \\
\hline & $\begin{array}{c}\text { Buena Opción de grado, conoces otras mane- } \\
\text { ras de investigar }\end{array}$ & 15 & $15 \%$ \\
\hline & $\begin{array}{l}\text { Conocimiento cultural en todo el sentido de la } \\
\text { palabra }\end{array}$ & 10 & $10 \%$ \\
\hline & $\begin{array}{c}\text { Es bueno que mejoren el sistema que tienen } \\
\text { para aumentar cantidad y calidad en investiga- } \\
\text { ciones }\end{array}$ & 15 & $15 \%$ \\
\hline & TOTAL & 100 & $100 \%$ \\
\hline
\end{tabular}

Tabla 10. Experiencia al representar a la Institución Fuente: elaboración propia 


\section{Resultados}

Con base en la información suministrada por la encuesta realizada a docentes y estudiantes de los programas académicos como negocios internacionales, contaduría pública, administración de empresas, gastronomía e ingeniería de telecomunicaciones de la Fundación Universitaria San Mateo, se pudo identificar lo siguiente:

1. Participan en la encuesta cinco programas académicos, que estuvieron abiertos a la convocatoria realizada.

2. Dentro de las respuestas que se obtienen la mayoría de los estudiantes encuestados no ubican la oficina del CDTEC.

3. De igual manera, la mayoría de estudiantes no conocen al director de la oficina del CDTEC y tampoco a sus colaboradores.

4. Con respecto a esta pregunta, en su mayoría, los estudiantes no conocen al coordinador de investigación del programa al que pertenecen.

5. Por ende no saben qué requisitos se necesitan para realizar investigación en la San Mateo.

6. De acuerdo con la respuesta anterior, el mayor porcentaje de los encuestados no han presentado proyectos de investigación ante el CDTEC.

7. Al no presentar proyectos, no tienen la posibilidad de participar en congresos de investigación. Un pequeño porcentaje de docentes lo ha hecho a nivel nacional e internacional.

8. En esta respuesta se observa que la mayoría de estudiantes y docentes no conocen el procedimiento para realizar movilidad en investigación en la Institución.

9. Estudiantes consideran que hacer investigación en la Institución le sirve para su área académica porque mejora el análisis e interpretación de la información, además de tener conocimientos más profundos sobre los temas a tratar; también porque mejora la calidad educativa y por último porque con esta opción pueden graduarse.

10. Se puede definir que la mayoría de los estudiantes no han tenido la experiencia de realizar movilidad investigativa; los que sí lo han hecho, dicen que es una excelente opción de grado porque perciben otras maneras de investigar; la experiencia es muy interesante, gratificante porque les amplía el conocimiento, además de relacionarse con otras culturas, consideran que se debe mejorar el sistema que se tiene para aumentar cantidad y calidad en las investigaciones.

Con lo expuesto anteriormente, respondiendo a la pregunta y objetivo general de la investigación se debe determinar manual de movilidad de estudiantes y docentes investigadores de la Fundación para la Educación Superior San Mateo (actualmente denominada Fundación Universitaria San Mateo), que debe ser de conocimiento general al público académico y administrativo, también se debe colgar a la página de la Institución.

Con lo anterior, se determina que requisitos, convocatorias, formularios y demás deben ser publicados de una manera clara y concisa en la página de la Institución, de esta forma se evita que estudiantes y docentes realicen antesala ante el CDTEC para que les expliquen los requisitos. La visita solo se debe dar para la entrega de documentos completos a la convocatoria postulada. 


\section{Conclusiones}

Para concluir la investigación, la Fundación para la Educación Superior San Mateo (actualmente denominada Fundación Universitaria San Mateo), debe tener en cuenta lo siguiente:

De acuerdo con el primer objetivo que es realizar diagnóstico de la situación actual sobre movilidad para docentes y estudiantes investigadores, se determina a través de la encuesta los inconvenientes que tienen docentes y estudiantes al aplicar a las convocatorias de movilidad investigativa ofertadas por el CDTEC.

Con el segundo objetivo que es identificar las necesidades en las que incurre la Fundación para la Educación Superior San Mateo (actualmente denominada Fundación Universitaria San Mateo), al realizar movilidad de estudiantes y docentes investigadores, se encuentran las siguientes: la oficina del CDTEC junto con sus colaboradores y coordinadores deben ser más visible dentro y fuera de la Institución. Difundir la necesidad de investigar y los beneficios que se obtienen al representar a la San Mateo en lugares diferentes al aula de clase. Incentivar la participación de estudiantes en las diversas convocatorias que existen. Divulgar constantemente a través de las redes sociales que maneja la San Mateo, información referente a congresos, eventos investigativos que puedan asistir como ponentes los docentes y estudiantes. Crear una página en Facebook para investigación que incluya pequeños resúmenes de lo que se hace mensualmente en los semilleros y eventos en los que se postule. Hacer listado de reconocimiento a los participantes y mostrar en las páginas al final de cada semestre académico.

Para el tercer objetivo que es generar estrategias, planes de acción para las buenas prácticas que debe implementar para movilidad, se debe hacer lo siguiente:

Semestralmente en el primer piso de la Institución el CDTEC debe realizar actividad para que el personal académico conozca lo que hace investigación, a través de pendones, recolección de solicitudes para postular proyectos e integrarlos a los semilleros de investigaciòn.

Realizar convocatoria de docentes y estudiantes que les guste investigar.

Hacer capacitaciones semestralmente para mejorar la calidad y cantidad en la producción de nuevo conocimiento. Cabe resaltar que la Institución busca constantemente cursos para que los docentes y estudiantes participen, de esta manera aumentar los estándares de calidad investigativa

Incentivar a que estudiantes se vinculen a los diferentes semilleros y grupos de investigación que actualmente hay en la San Mateo. Motivar para que al escoger la opción de grado, la investigación ocupe uno de los primeros lugares.

Al generar los grupos de investigadores, se debe dar a conocer los parámetros, normas y reglamento antes de iniciar las investigaciones, aunque estén en el sistema, existe la posibilidad que no las lean. 
Presentar y enseñar todo formato que se requiere para investigación.

Luego que la investigación finalice y cumpla con los parámetros exigidos, postular artículos en las diferentes revistas indexadas que de acuerdo al programa y temática puedan recibir calificación en Colciencias.

Postular los mejores trabajos investigativos para representación de la San Mateo a nivel local, nacional e internacional en los diferentes convenios que están actualmente vigentes.

En cuanto al tercer objetivo, elaborar manual de reglamentación de movilidad investigación: se debe analizar la situación con el CDTEC, solicitar los documentos necesarios para la elaboración del mismo, recibir opinión y aceptación por su parte, para que en el segundo semestre del 2018, esté el documento listo y alcance de todo el personal académico y administrativo de la Institución.

Con respecto a lo expuesto por la Universidad Distrital, se debe tomar como ejemplo la manera en la que promueven y difunde la información de movilidad tanto académica como investigativa. Las dos instituciones manejan números diferentes de estudiantes, pero es importante que la San Mateo analice la manera de publicar sus comunicados, es entendible que en el mundo actual, las personas dedican mucho más tiempo a las redes sociales que a los correos Institucionales o personales.

También debe buscar y ofertar convocatorias en las diferentes plazas que hay, aprovechar los convenios con los que se cuenta para realizar intercambios de corta, mediana y larga duración tanto para estudiantes como para docentes investigadores.

Bibliografía

Fundación para la Educaciòn Superior San Mateo. (2012). Estatuto de Investigaciones. Estatuto de Investigacion, Fundación para la Educacion Superior San Mateo, Centro de Desarrollo Tecnologico, Bogotá. Hernández Sampieri, R.; Fernández Collado, C.; y Baptista Lucio, M. (2014). Metodología de la investigación (6ta ed.). México. D.F.: McGraw-Hill Education/ Interamericana Editores S.A. de C.V.

Ministerio de Educación Nacional. (s.f.). Recuperado NA. (15 de 10 de 2017). Consejo Nacional de Acreditaciòn. Obtenido de https://www.cna.gov.co/1741/article-187351.html

SNA. (12 de 10 de 2017). Sistema Nacional de Acreditación en Colombia. Obtenido de https://www.cna.gov. co/1741/article-186365.html

COLCIENCIAS. (12 de 10 de 2017). Obtenido de www. colciencias.gov.co/colciencias/sobre_colciencias/acerca
Universidad Distrital Francisco José de Caldas. (s.f.). Recuperado de Universidad Distrital Francisco José de Caldas: www.udistrital.edu.co/historia

Secretarìa de Cultura, Recreación y Deporte. (28 de 04 de 2018). Recuperado de Bogotanitos: culturarecreacionydeporte.gov.co/es/bogotanitos/bogodatos/bogota-y-sus-localidades

UNIVERSIA. (11 de 10 de 2007). Obtenido de http:// noticias.universia.edu.pe/movilidad-academica/noticia/2007/10/11/741021/importancia-experiencia-intercambio-estudiantil.html

Ministerio de Educación Nacional. (s.f.). Recuperado de Ministerio de Educación Nacional: www.mineducacion. gov.co/1621/w3-propertyvalue-48391.html 